\title{
The Study of Non-Contact Anthropometry Technique and Method in Natural Wearing State
}

\author{
KeKe Sun ${ }^{1, a}$ and YiJui Chiu ${ }^{2, b}$ \\ ${ }^{1}$ School of Design Arts and Fashion Design, Xiamen University of Technology, No. 600, Ligong Rd, \\ Xiamen, 361024, Fujian Province, China. \\ ${ }^{2}$ School of Mechanical and Automotive Engineering, Xiamen University of Technology, No. 600, \\ Ligong Rd, Xiamen, 361024, Fujian Province, China. \\ asunkeke@xmut.edu.cn, ${ }^{b}$ chiuyijui@xmut.edu.cn
}

Keywords: B-spline; Anthropometry; Experiment; Curve fitting; Body silhouette

\begin{abstract}
This paper discusses how to get the body contour and size measurement, when people are measured wearing daily clothes in natural environment. This paper aims to propose the method of curve segmentation, extraction of effective feature points and curve fitting to make body silhouette curves reappear on the premise of somatotype, gets the rule of the body silhouette curves through contrasts between the fitting curves and the body silhouettes. And, calculate the final size in the garment design and manufacture, and puts forward some suggestions and put the attitude measurement. Being different from other 3D non-contact measuring methods, this paper provides a new way to get the BWH measurements in the condition of daily natural wearing, which not only optimize the non-contact anthropometrics theory, but also break the static measuring mode. Furthermore, the bringing forth of the concept of 'natural wearing state' and the laboratory experiments of this paper would be worthy for practical use.
\end{abstract}

\section{Introduction}

As an important branch of garment ergonomic, body measurement is the groundwork ensuring clothing design and manufacturing. The technique of non-contact body measurement gradually developed, which can offer accurate and full-scale measured data, to meet the demands of modern garment digital design and production. Over the past three decades, a number of non-contact body measurement system were developed in countries which have well-developed fashion industry. There are two major kinds of them, three dimensional and two dimensional. For 3D measurement systems, the white-light interference measuring system designed by American $\mathrm{TC}^{2}[1]$, and Vitus Smart 3D laser human body scanner developed by German TecMath and French Lectra [2][3][4], were representative. For 2D, Contour 2D system of German TecMath and BoSS-21 of Canadian VISMAGE SYSTEM should be mentioned.

And some researches focused on 2D and 2D-3D non-contact measurement, such as Gu et. al. [5] developed automatic pattern generation system of men's pants, which is based on two dimensional non-contact human body measurement systems. Guo et. al. [6] explored the application of the automatic clipping method in the 2Dimensional non-contact measuring system. Pirre and Shi [7] developed 2D image measurement system, which could determine the size of clothing. They found image-based systems are capable of providing anthropometric measurements, both in terms of accuracy and repeatability, which are better than traditional measurement methods. Yu et. al. [8] adopted a wide coverage of optical design. This system could obtain high resolution images in a short distance, and through the computer program into a 3D human body model. Kurita [9-10] developed a method for measurement of human motion, it is the electrostatic induction current non-contact type walking motion detection and non connection conditions based on the proposed technique is based on the signal detection, walking, estimation can be successful applied to human activities.

Based on the demands of measurement locations and accuracy in fashion industry, this project is aimed at the acquirement of measurements such as bust, waist and hips when people are measured 
wearing daily clothes in natural environment, solving the current ubiquitous problem of dress restraint.

\section{Analysis}

Through analysis of torso silhouette curves, we segment these curves regularly and do curve-fitting experiments according to the shape and trend of each segment, analyze the fitting results between fitting curves and real body curves, and then confirm the fitting functions. Thus, combining the data information of effective feature points in digital images of people measured in natural wearing state, torso silhouette curves reappear preliminarily. The research process and route is shown in the Fig. 1.

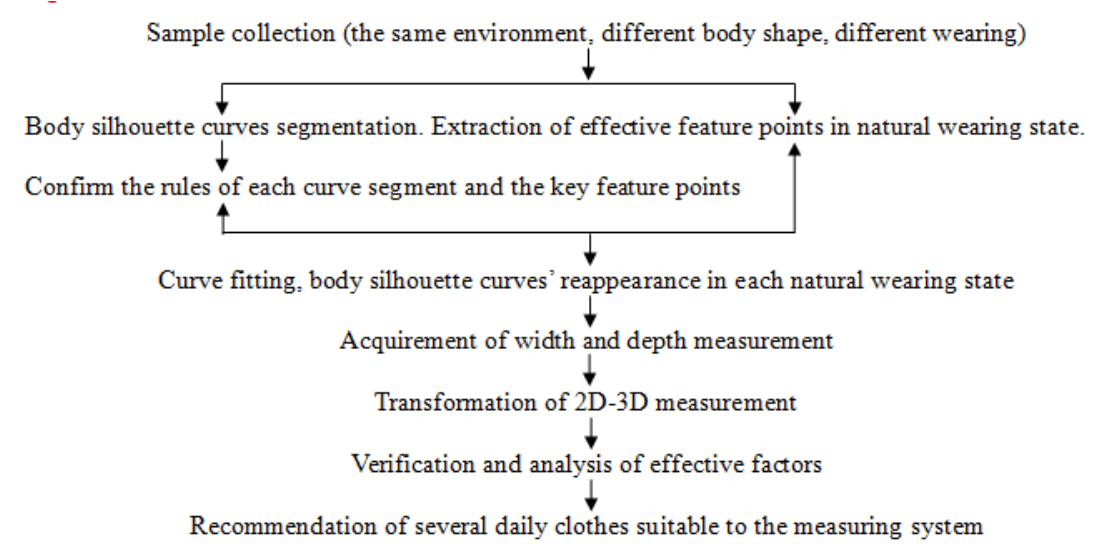

Figure 1. Research process

According to the continuous and smooth characteristic of body silhouette curves, we use the fitting function whose curve has good smoothness-B spline. It's mathematical model, as follows:

$\mathrm{B}-$ Spline curve equation is expressed as

$$
C(u)=\sum_{i=0}^{n} N_{i, p}(u) P_{i}
$$

Where $P_{i}$ is control points; $n+1$ is number of control points; $N_{i, p}(u)$ is basis function; $u$ is parameter; $p$ is degree.

The basis function is defined as:

$$
\begin{aligned}
& N_{i, o}(u)=\left\{\begin{array}{l}
0, u_{i} \leq u \leq u_{i+1} \\
1, \text { others }
\end{array}\right. \\
& N_{i, p}(u)=\frac{u-u_{i}}{u_{i+p}-u_{i}} N_{i, p-1}(u)+\frac{u_{i+p+1}-u}{u_{i+p+1}-u_{i+1}} N_{i+1, p-1}(u)
\end{aligned}
$$

B-Spline properties:

$$
\begin{aligned}
& \text { a. } N_{i, p}(u)=\left\{\begin{array}{l}
\geq 0, u \in\left[u_{i}, u_{i+p}\right] \\
=0, \text { others }
\end{array}\right. \\
& \text { b. } \sum_{i=0}^{n} N_{i, p}(u)=1, \quad u \in\left[u_{p-1}, u_{n+1}\right] \\
& \text { c. } \quad N_{i, p}(u)=\frac{k-1}{u_{i+p-1}-u_{i}} N_{i, p-1}(u)+\frac{k-1}{u_{i+p}-u_{i+1}} N_{i+1, p-1}(u)
\end{aligned}
$$

\section{Experiment}

Taking the body's front view and side view is the basis of getting the body two-dimensional measurements. This system takes digital images using ordinary digital camera, inexpensive, high 
precision, easy to deal with graphics.

a. Basic information of the measured people

(1) age range: 18 26(years). (2) somatotype: the thin, the normal, the fat, as shown in Table.1.

Evaluating human body shape, commonly used methods are: circumference difference, Rorel index, Verveck index, TNF index, bust/height, etc. According to the research of Kyoto Women's University in Japan, Verveck index is more a reflection of the Asian's body shape in thin or fat characteristic, than Rorel index. And for young bodies, TNF index is better. TNF index is also known as body linear density index, the formula of which is shown as follows:

$\mathrm{TNF}=\mathrm{W} / \mathrm{L} * 100$,

In which, W-weight $(\mathrm{kg})$; L-length $(\mathrm{cm})$.

Table.1. Somatotype criterion

\begin{tabular}{|c|c|c|c|}
\hline index $\quad$ somatotype & Thin & Normal & Plump \\
\hline TNF & $<32$ & $32-40$ & $>40$ \\
\hline
\end{tabular}

In addition, to the same measured person, we take the front view and side view when wearing tights, besides wearing several daily clothes, for use as comparisons. (3) dress classification:

(1) shirt+ pants (jeans) (2) T-shirt+ tailored skirt (A-line skirt) (3) sweater+ jeans (casual pants)

b. Major equipments (hardware): three dimensional calibration frame, fixable tripod, digital camera -"OLYMPUS C2500L", electronic computer, image acquisition card, etc.

c. Indoor environment: room temperature: $260 \mathrm{C}$, brightness: ordinary fluorescent lamplight.

d. Measuring method:

Take digital images of body's front view and side view using the digital camera. When taking front view, the measured people should stand on the fixed mark in the calibration frame, respectively wearing black elastic camisole and pants and various daily clothes, with their feet shoulder-width apart, arms opening 40o angle from body side, hair bundled up, underarm points bared, crotch points bared. When taking side view, the measured people should stand on the other fixed mark with their feet shoulder-width apart, arms clinging to body, exposing the whole front and back silhouette curves. Fix the tripod 2.5 meters right ahead the calibration frame, adjust and fix each performance index of the digital camera, such as aperture size, shutter speed, etc. Take pictures of front view and side view in sequence and finish storage images.

\section{Numerical Results}

On the basis of the mathematic form and experiment method shown in the two sections above, this section will illustrate the results of comparison between the fitting curves and the body's silhouette curves in front view (see Fig.2-Fig.4) and side view (see Fig.5-Fig.7). For lack of space, we only show the Normal here.

\section{Curve Fitting Comparison of Front Body}

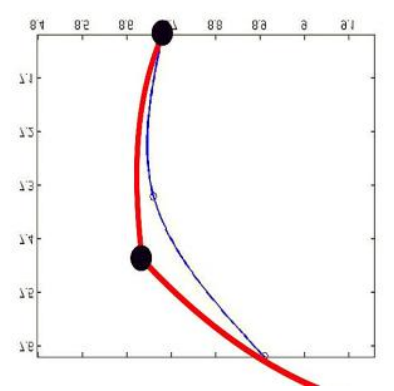

(a) Neck

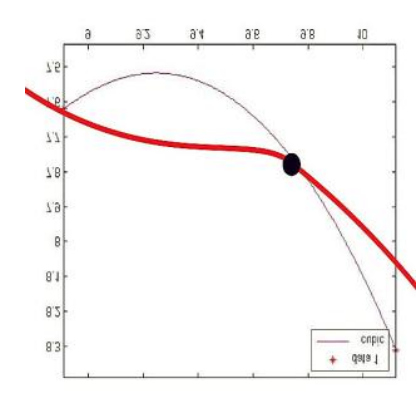

(b) Shoulder

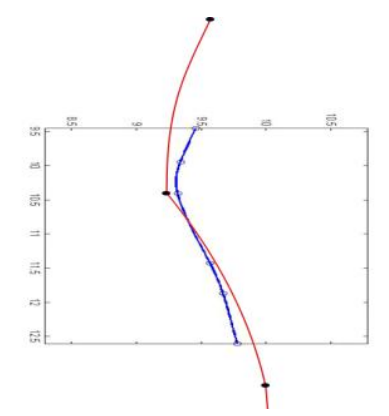

(c) Torso

Figure 2. Springand Autumn wearing (shirt+ pants) 


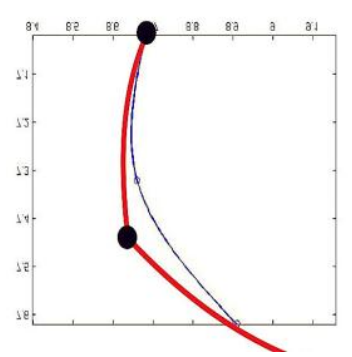

(a) Neck

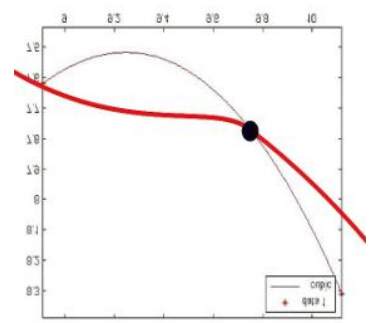

(b) Shoulder

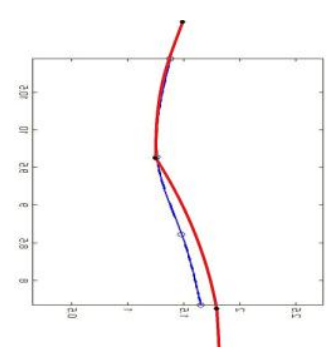

(c) Torso

Figure 3. Summer wearing (T-shirt+ tailored skirt)

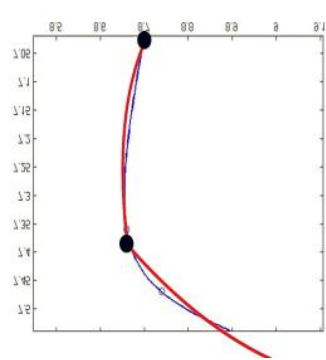

(a) Neck

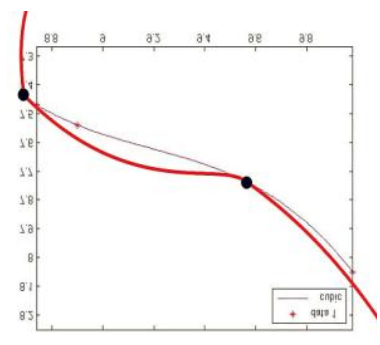

(b) Shoulder

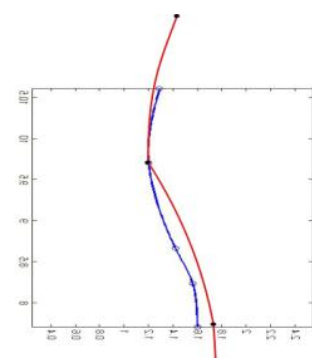

(c) Torso

Figure 4. Winter wearing (sweater+ casual pants)

As shown in Fig.2 - Fig.4, errors in shoulder measuring are large, next is neck, smaller in torso, but all have significant errors.

\section{Curve Fitting Comparison of Side Body}

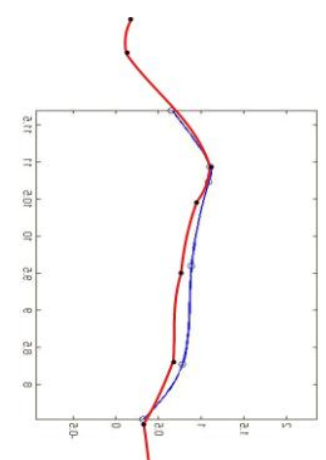

(a).chest side

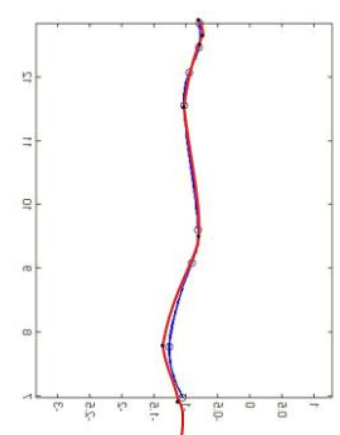

(b).dorsal part

Figure 5. Springand Autumn wearing (shirt+ pants).

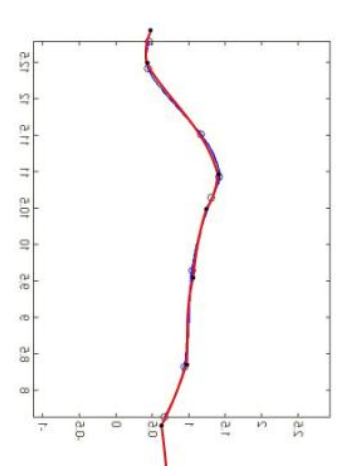

(a).chest side

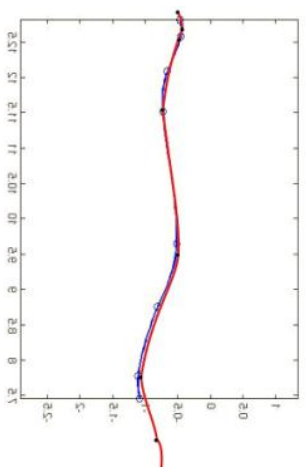

(b).dorsal part

Figure 6. Summer wearing (T-shirt+ tailored skirt). 


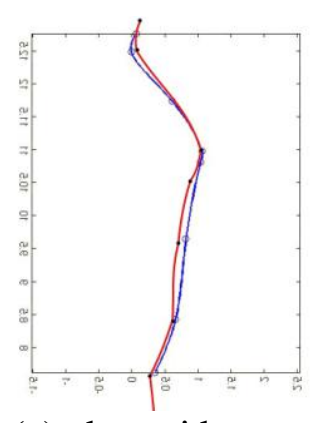

(a).chest side

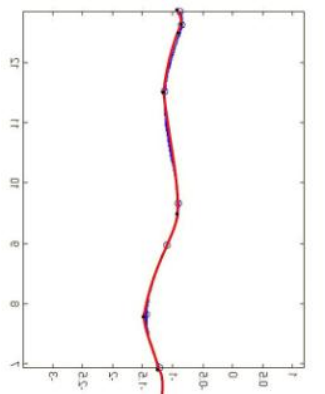

(b).dorsal part

Figure 7. Winter wearing (sweater+ casual pants)

As shown in Fig.5-Fig.7, errors in chest side are bigger than in dorsal part, but all smaller than front body.

\section{Reappearance of Torso Silhouette Curves in the Natural Wearing State}

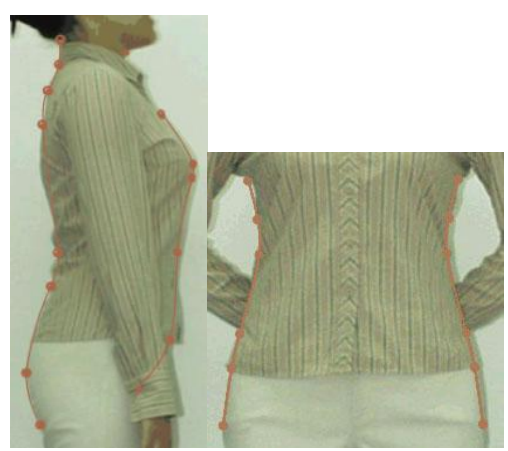

(1) Springand Autumn wearing

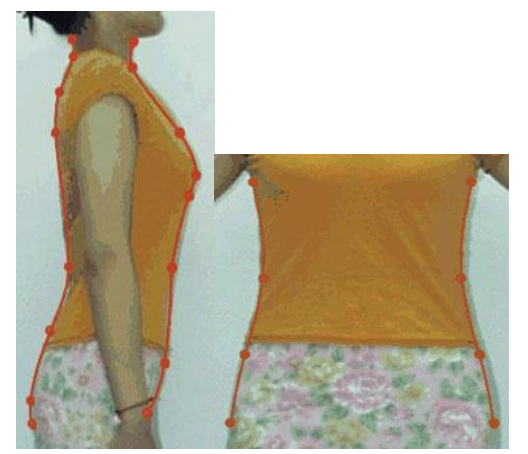

(2) Summer

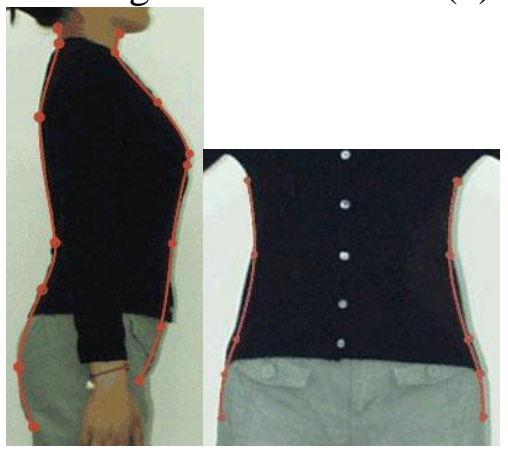

(3) Winter wearing

Figure 8. Reappearance of torso silhouette curves in the natural wearing state

According to the fitting curves in Fig.8, we can directly measured the segment length of chest( waist, hip) width and depth, with the human body cross-section views in chest, waist and hip, and 2D-3D transformation method, measurements are worked out.

\section{Error Analysis}

Throughout the comparison figures, we qualitatively analyze every part from photographing to fitting, summarize several procreant reasons of error as follows:

(1) The body posture and breath state of the same measured person wearing tights and daily clothes aren't completely same. There must be difference in original states when taking digital images of different wearing.

(2) Judging each key feature point and getting its data information are manually operated, there must be some personal errors.

(3) Fitting method itself is a process of approximate treatment, also a source of error.

Because of inevitable objective and subjective errors, the fitting results can't be identical with the body, but if they can fully meet the accuracy demands of body measuring for clothes design and 
producing, it remains positive and worthy of market promotional application.

\section{Conclusion}

The research concludes as follows:

(1) Propose the research idea of "in natural wearing state, through image information processing, take the steps of 'curve segmentation - extraction of effective feature points - curve fitting', to make body silhouette curves reappear".

(2) The measured samples' somatotype is the premise of the analysis of curve shape rules. Determine the thinking of "somatotype before curve fitting", and with experimental verification, the fitting results are good, which make the research of body silhouette curves deeper.

(3) By analyzing image effects, preliminarily confirm a number of daily clothes types and postures for body measurement, simple and easy to operate, provide basic technical support for further development of measuring system.

(4) Realize the development from wearing tights to daily dress, make the measuring system more convenient and humanistic.

\section{Recommendation}

We suggest that measured people has better not wear outer garment, such as windbreakers and overcoats, they can be measured in several common everyday wear by seasons:

Spring and Autumn: Top, fit light sweaters or shirts are recommended, whose fabrics are soft and draping, such as thin polyester-cotton. Bottom, jeans and pants, fit in hip, because the hip measurement does not require leg curves.

Summer: Top, common T-shirts can meet the measuring demands, whether formal or casual. Besides, sleeveless blouses or dresses women usually wear in summer to highlight their figure advantages, are advisable. Bottom, skirts are in the majority, tailored skirt is recommended, circular skirt and umbrella skirt which have fullness in hip are not.

Winter: Top, common sweaters or thermal underwear are recommended, bottom, pretty much the same as spring and summer.

\section{Acknowledgement}

All the research works are supported by Fujian Nature foundation (2016J01039), Xiamen University of Technology talent foundation (YKJ15027R), Fujian Social Science Project (FJ2016C118), Fujian Provincial Department of Education Project (JAS150434)

\section{References}

[1] Hilton, D. Beresford, The Visual Computer, Vol.16 (2000), p.411-436.

[2] N. D'apuzzo, Journal of Photogrammetry and Remote Sensing, Vol. 56 (2002), p.360-375.

[3] S. Thomas, B. Jochen, B. Heiner, International Journal of Industrial Ergonomics, Vol. 25 (2000), p.447-453.

[4] The complete process of mass customization, http://www.lectra.com.cn.

[5] Gu, B.F., Kong, H.Y., Gu, P.Y., Su, J.Q., Liu, G.L. (2011), Proceedings - 2011 International Conference on Future Computer Sciences and Application, pp. 150-153.

[6] Guo, P.P., Liu, G.L., Dai, H.Q. (2015), Textile Bioengineering and Informatics Symposium Proceedings 2015, pp. 589-595.

[7] Pirre, M., Shi Y., (2000), Applied Ergonomics, 31, pp.445-451.

[8] Yu, W., Ng R., and Yan S., (2002), Journal of HongKong Polytechnic University, 28, 55-62. 
[9] Kurita, K., (2011), Measurement: Journal of the International Measurement Confederation. 44 (8), pp. 1361-1366.

[10] Kurita, K., (2014), 11th IMEKO TC14 Symposium on Laser Metrology for Precision Measurement and Inspection in Industry, pp. 82-85. 